\title{
Perspectiva de género en el sistema educativo según la percepción de los estudiantes de educación superior
}

\author{
Gender perspective in the educational system according to the perception \\ of higher education students
}

\author{
Armando Ortiz Aragón \\ Instituto de Ciencias Sociales Universidad Juárez del Estado de Durango \\ aortiza@ujed.mx \\ Fabiana Esther Mollinedo Montaño \\ Universidad Autónoma de Zacatecas "Francisco García Salinas" \\ fabiana.mollinedo@uaz.edu.mx
}

\begin{abstract}
Resumen
La perspectiva de género a iniciativa del Instituto Nacional de las Mujeres y con el impulso de la Secretaria de Educación se aplica en el sistema educativo considerando la escuela como un efectivo medio de trasmisión de valores, un espacio de sociabilización con reglas que moldean al individuo, caracterizado por el hecho de que aún sin proponérselo tiende a reproducir el sistema social existente. Las Instituciones pertenecientes al sistema de educación pueden recibir por parte del INMUJERES la certificación en el Modelo de Equidad de Género (MEG) cuando incorporan estrategias de género en sus políticas internas e instrumentan acciones afirmativas. Con el objetivo de identificar cómo consideran los estudiantes de licenciatura que la perspectiva de género influye en su desarrollo académico y humano, así como la valoración de la importancia que otorgan a las acciones afirmativas de género llevadas por parte de la administración, se realizó una investigación en la Universidad Juárez del Estado de Durango (UJED) y el Instituto Tecnológico de Durango (ITD). Como principales resultados se tiene que los encuestados consideran que el desempeño académico está en función de sus condiciones personales, que su trato con la diversidad y la equidad de género en las actividades académicas son producto de su propia decisión de convivencia y no de acciones afirmativas de la normatividad institucional, además de que las decisiones de la administración, como el caso de la poca flexibilidad en los horarios de estudio, no contribuyen a evitar problemas como la deserción o a resolver dificultades extra académicas.
\end{abstract}

\section{Palabras clave}

Perspectiva de género, educación superior, desarrollo académico y humano.

\begin{abstract}
The gender perspective at the initiative of the National Institute for Women and with the encouragement of the Ministry of Education is applied in the education system considering the school as an effective means of transmitting values, a space of socialization with rules that shape the individual, characterized by the fact that even unintentionally it tends to mimic the existing
\end{abstract}


social system. Institutions belonging to the education system can receive certification from the INMUJERES in the Gender Equality Model (GEM) when they incorporate gender strategies into their internal policies and implement affirmative actions. With the objective of identifying how the undergraduate students consider that the gender perspective influences their academic and human development, as well as the assessment of the importance they give to the affirmative gender actions taken by the administration, an investigation was carried out. at the Juárez University of the State of Durango (JUSD) and the Technological Institute of Durango (TID). The main results share that respondents consider that academic performance is a function of their personal conditions, their treatment of diversity and gender equality in academic activities is the product of their own decision to live together and not that of affirmative action. The decisions of the administration, like cases of little flexibility regarding class schedule, do not contribute to avoid problems like desertion or to solve extra academic difficulties.

\section{Keywords}

Gender perspective, higher education, academic and human development.

\section{Introducción}

Las instituciones de Educación superior, ante las iniciativas de organismos internacionales y las experiencias de Universidades de otras regiones realizan desde la década de los 70's aportaciones teóricas y metodológicas encaminadas a eliminar la brecha propiciada por la inequidad sobre todo en el aspecto referente al género.

Por otra parte, las acciones de los Gobiernos relativas a este aspecto son orientadas por acuerdos tomados de Convenciones promovidas por la Asamblea General de las Naciones Unidas como es la Convención sobre la eliminación de todas las formas de discriminación contra la mujer (CEDAW) a la que México se adhirió en 1981.

La escuela o el ámbito educativo es un efectivo medio de trasmisión de valores, después de la familia misma o el entorno inmediato del ser humano. Sabiendo que el sistema educativo es entonces más que un ente expendedor de títulos, un espacio de sociabilización con reglas que moldean al individuo y además caracterizado por el hecho de que sin proponérselo tiende a reproducir el sistema social existente, ha propiciado que se confine o subestime el papel de la mujer en la sociedad, limitando seriamente su desarrollo humano y forzándola a adaptarse a patrones que de manera sutil le orientan hacia la elección de carreras profesionales acordes con su género aunque esta elección no siempre corresponda a sus deseos o capacidades.

Es tal la influencia de la división genérica de profesiones que incluso llega a suceder que la sociedad no valore o lo haga de forma condicionada y limitada a las profesiones y oficios que desempeña la mujer. Este fenómeno es de tal magnitud que se ha percibido que aquellas disciplinas que se feminizan, de forma dramática pierden posicionamiento en el reconocimiento social, pasando a ser de estatus inferior porque las realizan las mujeres.

Es importante recalcar que en un principio se consideraba exitoso cualquier programa encaminado a disipar la inequidad de género cuando cuantitativamente se lograba incrementar la incorporación de las mujeres a las oportunidades de educación, pero pronto se puso de manifiesto que no se tomaba en cuenta aspectos cualitativos como los factores socioculturales o como la oferta educativa inciden en el desarrollo humano de las mujeres. 
Al respecto de esta problemática, circunscribiéndola a la educación superior, Buquet $^{1}$ enfatiza sobre los problemas conceptuales y prácticos de la transversalización de la perspectiva de género en la educación superior y señala tres vertientes destacables dentro del ámbito de estudios de género: la investigación, la formación y la institucionalización de la equidad de Género.

En dicha propuesta Buquet señala algunas acciones que deben ser emprendidas por las Universidades para transformar positivamente las relaciones de género al interior de las mismas, a sabiendas que sus efectos permearán hacia la sociedad por efecto de las acciones de sus egresados.

\section{Planteamiento de la investigación}

Ante lo anterior se establece la importancia de indagar aspectos ocultos dentro de sistema educativo que esté contribuyendo a la inequidad señalada y la percepción que de estos tienen los propios estudiantes.

\section{Objetivos}

1. Mostrar si la perspectiva de género influye en el desarrollo humano y desarrollo académico en las instituciones de educación superior

2. Identificar la percepción de los estudiantes respecto del acoso sexual y la violencia de género.

3. Describir las situaciones que los estudiantes consideran más importantes en su desenvolvimiento dentro de las instituciones de educación superior.
4. Caracterizar las condiciones que los estudiantes tienen para su desarrollo académico.

5. Establecer el significado que tiene el desarrollo humano para los estudiantes de educación superior.

\section{Referentes teoricos}

La problemática de la inequidad de género como resultado de una cultura patriarcal permea a todos los ámbitos de la actividad humana. Las Instituciones de Educación Superior son transmisoras de estos estereotipos a través de su currículo y en la convivencia y relaciones entre profesore(a)s y alumno(a)s, lo cual se manifiesta de forma evidente y velada a la vez dentro de sistema que contribuye a la inequidad señalada.

En lo que concierne a la educación superior que es impartida en instituciones tanto públicas como privadas, se ha establecido una agenda encaminada a la equidad de género, las acciones positivas consisten, según señala González (2013), en involucrar a las Universidades para que dentro de las aulas se fomente el trato respetuoso y que de todas las escuelas surjan estudios con enfoque de género que lleguen a ser propuestas para políticas públicas.

El principio de equidad derivado de la igualdad de oportunidades enmascara una realidad que en el caso de la educación superior queda al descubierto cuando según señala Arenas (2013) en lo que a derecho a la educación concierne $\mathrm{y}$ al trato no discriminatorio en esta materia... es necesario abordarla en dos perspectivas, la primera el ingreso al sistema educativo y la segunda la permanencia, si bien cuentan las mujeres y los hombres con trato digno e igualitario para

\footnotetext{
1 Secretaria de Equidad de Género del Programa Universitario de Estudios de Género de la UNAM. Estudiante de doctorado en Sociología en la UNAM, licenciada y maestra en Psicología; especializada en género, sexualidad y educación.
} 
acceder a la educación, también debemos de considerar que las condiciones de permanencia no necesariamente son igualitarias... de acuerdo con la encuesta nacional de la juventud 2010 el $75.7 \%$ de los jóvenes que ni estudian ni trabajan a nivel nacional son mujeres, siendo las principales causas por las que abandonan su formación el matrimonio, la situación económica o por razones académicas".

Buquet (2010) marca de manera concreta que la presencia de las mujeres en las Universidades no es sinónimo de equidad de género.

\section{Metodologia}

El estudio fue de tipo exploratorio, con un muestreo aleatorio simple se consideró una muestra de 445 sujetos de ambos sexos Con una distribución proporcional en la aplicación del instrumento: UJED con 9,707 alumnos, equivalentes al $62 \%$ se proyectó aplicar 262 encuestas e ITD con 6,002 alumnos, equivalentes al 38\% se proyectó aplicar 183 encuestas, durante el semestre 2014A. Se procedió enseguida a elegir las escuelas a visitar fundamentado primero en una clasificación por DES (Dependencia de educación superior) en el caso de la Universidad y por Departamento Académico en el caso del Tecnológico, se eligieron utilizando una tabla de números aleatorios las carreras a las que se aplicaría la encuesta, teniéndolas ordenadas de acuerdo con la forma que se encuentran enlistadas en las páginas web oficiales de las Instituciones (consultadas el 28 de mayo de 2014).

El procedimiento se efectuó de la siguiente manera:

- Al elegir el programa académico por institución se contabilizo la cantidad de alumnos inscritos y se determinó su aportación a la proporción correspondiente

Ortiz Aragón, A.; y Mollinedo Montaño, F.E.
- Se obtuvo la diferencia respecto a la proporción esperada

- El valor obtenido se evaluó para justificar si se continuaba con otra selección o se detenía el proceso.

- Los pasos se repitieron hasta que la proporción correspondiente por institución se alcanzó y la diferencia después de mostrar un crecimiento comenzó a declinar llegando a ser menor al $10 \%$.

Para la recolección de datos se utilizó un cuestionario conformado por 27 variables nominales con una escala dicotómica y politómicas y 123 variables ordinales, con una escala de 0 al 10 donde el 0 es ausencia total del atributo y el 10 la expresión máxima del mismo.

Las carreras por institución que fueron elegidas una a la vez de forma alternada y secuencial según la lista obtenida en el proceso aleatorio para aplicar el instrumento de investigación. En la Universidad Juárez Del Estado De Durango, las carreras de Contador Público 6.52 \% (29); Administración 8.31\% (37); Psicología 8.76 \% (39); Pintura y escultura $4.72 \%$ (21); Matemáticas $2.47 \%$ (11); Ciencias Químicas 6.29\% (28); Ciencias Forestales 10.79 \% (48); Trabajo Social 6.52 \% (29); Educación Física 4.49 \% (20) haciendo un porcentaje de $58.87 \%$ (262), de la misma manera en el Instituto Tecnológico De Durango, Administración 6.52 \% (55); Ingeniería Industrial 8.31\% (30); Gestión empresarial $8.76 \%$ (28); Ingeniería Civil 4.72 \% (25); Ingeniería Mecatrónica 2.47\% (45) haciendo un subtotal de $41.12 \%$ (183) que en un $100 \%$ son (445).

\section{Resultados de investigación}

Con la finalidad de evaluar la consistencia interna del instrumento de investigación se utilizó el Coeficiente Alfa de Cronbach, obteniéndose $\alpha=0.91$ normal, que indicó que se trató de un instrumento fiable, que hace 
mediciones estables y consistentes, donde el error estándar es menor al 5\%. No se encontraron reactivos redundantes mayores a 0.96 .

Con respecto a la edad de los sujetos participantes, se encuentra que un 48\% (215) se encuentra entre 21 a 23 años, un 46\% (205) entre 18 a 20 años, asi como tan solo un 6\% (25) se encuentra entre 29 a 34 años. El semestre con mayor representación fue el 2 con un $21.57 \%$ seguido del sexto semestre con $16.85 \%$.

Respecto al promedio académico del semestre anterior se observa que un $48.99 \%$ (218) tiene un promedio entre 81 a 90; un $23.37 \%$ (104) tiene un promedio entre $91 \mathrm{a}$ 100 y un 21\%(96) tiene un promedio de 71 a 80 , tan solo un $0.67 \%$ (3) tienen un promedio entre 60 a 70.

Para el análisis de comparaciones se utilizó el estadígrafo paramétrico t de Student, donde los niveles de probabilidad son de $\mathrm{p} \leq$ 0.05 , con el objeto de explicar la contrastación en las respuestas de los sujetos en sus diversas características.

Al comparar las variables nominales por sexo de los encuestados se encontró que existe una gran similitud entre las medias de ambos y solo hay una diferencia significativa en lo que corresponde a las miradas morbosas o gestos sugestivos que molestan, donde la media de los hombres es de 1.58 y la de las mujeres de 2.80, arrojando estos valores que la importancia a tal situación se le otorga en mayor medida por parte de las mujeres y que es significativa la divergencia.

La comparación de las variables nominales por Institución Educativa en la que los encuestados están cursando su licenciatura muestra diferencia significativa en lo que corresponde a la oportunidad que consideran tener los estudiantes de poder seleccionar su horario de clases, los inscritos en el Instituto Tecnológico de Durango con 7.6 y aquellos que están en la Universidad Juárez del Estado de Durango con 3.9; en lo que atañe a las investigaciones promovidas por parte de las Autoridades del plantel respecto de eventos de acoso o violencia sexual la diferencia significativa arrojada por la prueba t pone de manifiesto con una media para el Instituto Tecnológico de 3.5 y de 2.4 para la Universidad Juárez, lo que pone en claro que la respuesta oficial al acoso sexual se da en mayor medida en el ITD que en la UJED.

El análisis factorial en modalidad de Comunalidades arroja 14 factores, que explican el 53\% de la variabilidad total del fenómeno con un Eigen value mayor a 1. Las cargas factoriales aceptadas de acuerdo al tamaño de la muestra, obtenidas por el cálculo de probabilidades del programa estadístico fue de 0.12 .

La lectura de los resultados da cuenta de cómo los encuestados consideran que el desempeño académico está directamente relacionado con las actividades que realizan en su hogar, las necesidades económicas y el estado de salud. Por otra parte se puede observar que las condiciones que ofrece la institución de educación no contribuyen a favorecer el tiempo disponible para estudiar, no evitan la deserción, no se reconoce en la magnitud deseable el trabajo realizado y razonan que no reciben apoyo en problemas extra académicos.

A pesar de lo anterior se ve como positivo el ambiente de las instituciones dado que se muestra que los entrevistados tienen libertades que inciden en la posibilidad de independencia de pensamiento, emociones, identidad sexual, igualdad de derechos colectivos e independientes del sexo y la inexistencia de roles impuestos por tradición. Y respecto de la violencia de género esta no parece ser una preocupación de los alumnos que expresan valores negativos para las condiciones relativas a estos aspectos, lo que arroja la lectura señalada al principio del párrafo. 
Queda de manifiesto que los entrevistados perciben libertades en cuanto a seleccionar horarios, participar en clase, opinar, liderar y trabajar en equipo, pero no denotan sentir que su trabajo sea valorado adecuadamente. En cuanto al acoso muestran indiferencia hacia los carteles o salvapantallas con connotación sexual y no les son molestos los comentarios que se dirijan hacia su apariencia cualquiera que sea el origen de los mismos profesores, compañeros o personal administrativo.

Por otro lado refrendan en sus respuestas la existencia de acoso en forma de roces o contacto físico, llamadas telefónicas o mensajes de naturaleza sexual, invitaciones forzadas y amenazas con afectar sus calificaciones en caso de no acceder al acoso por parte de los profesores hombres.

\section{Conclusiones}

La iniciativa del Gobierno Federal a través de la Secretaria de Educación Pública y el INMUJERES de hacer que la perspectiva de género en las instituciones de educación superior sea parte de la políticas internas de las mismas, dista de estar generando resultados satisfactorios debido a que se carece de un diagnostico que considere la percepción de los estudiantes respecto de lo que les es más relevante.

Si bien la perspectiva de género busca incidir en aspectos relativos al desempeño académico y humano de los educandos, estos últimos dan mayor relevancia a aspectos administrativos que limitan dicho desarrollo dejando incluso como algo secundario lo que corresponde a discriminación o incluso violencia de género.

Se observan libertades que son apreciadas por los estudiantes quienes las ven como un logro derivado más de la coexistencia entre ellos mismos que de las acciones institucionales, lo cual las hace multiplicarse en sus procesos de convivencia.

Ortiz Aragón, A.; y Mollinedo Montaño, F.E.
La obtención de la certificación en el Modelo de Equidad de Género (MEG) no es suficiente si es que se aplica de forma superficial y sin considerar que es un proceso permanente, pero sobre todo que sea generalizado, aceptado y puesto en marcha por la totalidad de la comunidad académica de las instituciones educativas y que permita atender las opiniones de quienes la conforman, aun de quienes en ocasiones son considerados subordinados al sistema, en este caso los estudiantes.

Se hace necesario un trabajo previo más amplio y comprometido que además considere la participación de los involucrados, para este caso en particular, los estudiantes, los docentes y el personal de apoyo, de tal forma que su aplicación no provenga solo de la administración y que al escuchar todas las voces se parta de un diagnostico pertinente para la misma.

Por otra parte es indispensable la capacitación, el conocimiento y la profundización en temas de género que emanen de las políticas en un proceso continuo y extendido.

\section{Referencias}

Barragan y Romero. (2014). La equidad hacia la mujer en la Universidad Pública, aportes para la construcción de la democracia. Universidad Juárez del Estado de Durango, Universidad Castilla-La Mancha.

Bonder, Gloria (1994). Mujer y educación en América Latina: hacia la igualdad de oportunidades. Revista Iberoamericana de educación. No. 6, sep-dic 1994. Biblioteca digital OEI.

Buquet Corleto, Ana. (2011). Trasversalización de la perspectiva de género en educación superior. Problemas conceptuales. Revista perfiles educativos. Vol. XXXIII, número especial, 2011. UNAM 
Bustos Romero, Olga. (2004). Reordenamientos genéricos de la matrícula en la educación superior. El caso de México. Revista Otras Miradas Grupo de Investigación en Género y Sexualidad GIGESEX. Vol. 1, No 4, Facultad de Humanidades y Educación Universidad de Los Andes, Mérida Venezuela.

Comité de estudios para el adelanto de las mujeres y la equidad de género, Centro de estudios para el adelanto de las mujeres y la equidad de género (2011). Violencia de género en México, estadísticas, marco jurídico, propuestas, políticas públicas. $\mathrm{H}$. Congreso de la Unión, Cámara de Diputados LXI legislatura. México.
Sánchez, Alma Rosa.(2003). Influjo de la división genérica de profesiones y oficios sobre la escolaridad y el empleo, en Escolaridad y trabajo femeninos en el contexto de la división genérica de profesiones y oficios. ENEP AcatlánUNAM, México.

SER/UNIFEM/PNUD. (2004) Manual: Convención sobre la eliminación de todas las formas de discriminación contra las mujeres y su protocolo facultativo CEDAW, $2^{\text {a }}$ edición, México.

Subirats, Marina.(2005). Género y escuela, en Construcción de identidades y género en la escuela secundaria. INMUJERES, México.

Valles y Corral. (2014). La presencia femenina en el Instituto Juárez. Instituto de Investigaciones históricas UJED. Durango, Mex. 
RECIE. Revista Electrónica Científica de Investigación Educativa Vol. 3, núm. 2, enero-diciembre 2017, pp. 885-891. 